\title{
Evaluation of Fracture Resistance Force in Three Types of Primary Molar Crowns: Milled by CAD|CAM, 3D Dental Printed, and Composite Celluloid Crowns
}

\author{
Mohammed Nour Al-Halabi ${ }^{1} \quad$ Nada Bshara ${ }^{1} \quad J o h n$ C. Comisi², $\quad$ Jihad Abou Nassar ${ }^{1}$ \\ 1 Pediatric Dentistry Department, Dental College, Damascus \\ University, Damascus, Syria \\ 2Department of Oral Rehabilitation, Medical University of South \\ Carolina, James B. Edwards College of Dental Medicine, Charleston, \\ South Carolina, United States \\ Address for correspondence John C. Comisi, DDS, Department of \\ Oral Rehabilitation, Medical University of South Carolina, \\ James B Edwards College of Dental Medicine, 173 Ashely Ave nue \\ BSB 548 MSC 507, Charleston, SC 29425, United States \\ (e-mail: comisi@musc.edu).
}

\begin{abstract}
Keywords

- computer-aided design/ computer-assisted milled crowns

- primary molars

- fracture resistance force

- stainless-steel crown

Objective This study compares three types of esthetic crowns in fracture resistance force (FRF) and failure type: (1) polymethylmethacrylate (PMMA) milled by computer-aided design/computer-assisted manufacture (CAD|CAM), (2) resin-based material via three-dimensional (3D) dental printer, and (3) direct composite celluloid crowns technique in primary molars.

Material and Methods Thirty lower second primary molar were randomized into three experimental groups consisting of ten molars for each: group A:CAD|CAM crowns using PMMA blocks; group B: 3D dental printer to fabricate crowns using glycidyl carbamate photopolymer resin; group C: Selected teeth crowned using direct resin composite celluloid crowns. The three groups (A, B, and C) were stored in water at $37^{\circ} \mathrm{C}$ for 30 days. The FRFs for the experimental crowned teeth were measured using a universal test machine (Testometric) until fracture and the values compared with the mean maximum bite force of children in the primary dentition. FRFs and failure types were recorded and statistically analyzed.

Results One-way analysis of variance (ANOVA) revealed significant differences among the groups in FRF testing. Bonferroni test was used for multiple-correction comparison in comparing force needed to fracture the specimens in the three groups. A significant difference was noticed between groups B-C $(p=0.000)$ and groups A-C $(p=0.000)$. No significant statistical differences were noted between groups $A-B(p=0.325)$ in FRF scores. In failure type, no statistical differences were noted when comparing groups, $A$ and $B$, groups $B$ and $C$, but comparing groups $A$ and $C$ showed statistical differences in chi-square statistical test.

Conclusion The three tested esthetic crowns exceeded the mean maximum bite force of children in the primary dentition; thus, they can be expected to perform well clinically. Even though using an indirect technique (CAD|CAM milling and 3D printer) showed a higher score in FRF, direct composite celluloid crowns technique can be beneficial since it is easier to repair and still is a useful modality.
\end{abstract}

(c) 2020. European Dental Research and Biomaterials Journal.

This is an open access article published by Thieme under the terms of the Creative Commons Attribution-NonDerivative-NonCommercial-License, permitting copying and reproduction so long as the original work is given appropriate credit. Contents may not be used for commercial purposes, or adapted, remixed, transformed or built upon. (https://creativecommons.org/licenses/by-nc-nd/4.0/) Thieme Medical and Scientific Publishers Pvt. Ltd., A-12, 2nd Floor, Sector 2, Noida-201301 UP, India 


\section{Introduction}

Trends for esthetics restorations have been grown dramatically, and this has become important to parents when the treatment of their children is concerned..$^{1,2}$

Managing severely carious primary molars is always a clinical challenge for pediatric dentists, especially in regards to aesthetic treatment due to limited treatment options. ${ }^{3}$ The use of stainless-steel crowns (SSCs) has been the treatment standard for extensive carious primary molars. SSCs have demonstrated long-term clinical performance, the ability to limit the secondary carries, cost-effectiveness, and ease of use. ${ }^{4}$ This preference is especially true for patient treatment when performed under sedation or general anesthesia. ${ }^{5.6}$ However, there is a growing demand by parents for treatments that have more esthetic options. ${ }^{1}$

The advances in adhesive dental dentistry have spilled over into the use of direct and indirect treatment options in the care of primary teeth. ${ }^{1.7}$ The use of composite crown-form restorations for severely decayed primary molars has been mentioned in the literature, ${ }^{8}$ and proper employment of these techniques could provide a long-term beneficial esthetic service and adequate substitute for traditional SSC restoration.

The improvements in digital dentistry, especially milling technology, have also contributed to this excitement for esthetic solutions in pediatric dentistry. This digital technology has evolved into two main fabricating procedures, computer-aided design/computer-aided manufacturing (CAD/CAM) milling system technology (subtractive manufacturing) and three-dimensional (3D) printing system technology (additive manufacturing). ${ }^{9} 10$ 3D printing, using newer printable dental material with physical properties to create viable dental restorations with sufficient mechanical properties for intraoral dental applications, ${ }^{11,12}$ has provided excitement in the restorative realm. Additionally, the various CAD/ CAM materials available to fabricate restorations via milling, can provide high-quality complete crowns ${ }^{13}$ including the use of durable polymethylmethacrylate (PMMA)-based polymeric resins ${ }^{14}$ This leads to greater possibilities when restoring primary posterior teeth.

To our knowledge, no studies have been conducted to compare the fracture resistance force (FRF) abilities of these materials when cemented or bonded to natural primary teeth. Therefore, the aim of this in vitro study was to evaluate the fracture resistance of three types of crowns: PMMA using CAD $\mid$ CAM milling, 3D printable photopolymer resin via 3D printer; and direct composite celluloid crowns technique to determine FRF (load-to-fractured test) and failure type to determine the potential for long-term clinical use for these types of crowns.

\section{Materials and Methods}

Thirty freshly extracted deidentified, intact, and sound second primary molars were collected in accordance to the ethical treatment of human tissue (IRB \#1191, Faculty of Dentistry, Damascus University) and were crowned with one of the three types of tested crowns according to their groups.
Group A: selected teeth crowned with polymethyl methacrylate (CopraTemp Symphony, PMMA)-based crowns fabricated using $C A D \mid C A M$; group $B$ : selected teeth crowned using 3D printable bio-compatible light-curing composite crowns (GC Temp PRINT; Tokyo, Japan) fabricated using a 3D dental printer (3D dental printer); group C: selected teeth crowned using direct composite celluloid crown technique.

The selected teeth in the three groups (A, B, and C) were stored in a solution saturated with thymol for 7 days, then rinsed and cleaned in distilled water prior to being embedded in acrylic resin $2.0 \mathrm{~mm}$ below the cementoenamel junction.

All samples were subsequently prepared for crowning with a diamond bur round end taper for buccal, lingual, mesial, and distal wall preparation of 0.8 to $1.0 \mathrm{~mm}$, followed by a chamfer margin circumferentially and occlusal reduction of 1.0 to $1.5 \mathrm{~mm}$ with a wheel no. 909 Komet, (Brasseler, Lemgo, Germany) with a convergence angle of 6 degrees. ${ }^{15}$ Polyvinylsiloxane impressions (Examix NDS; GC Corporation, Tokyo, Japan) were taken for each of the prepared teeth in their respective groups (A and B) that would receive the indirect restorations.

Celluloid crowns for group $C$ were selected to match the size of second lower primary molars using TDV celluloid crowns (TDV Dental; Pomerode, SC, Brazil). In group C, the celluloid crown matrices were trimmed to ensure proper placement and coverage of primary molars, and the prepared teeth were etched with $37 \%$ phosphoric acid and cleaned.

A bonding agent was applied to the tooth (Ivoclar Vivadent Tetric N-Bond) and cured for 20 seconds. The celluloid crowns were filled with resin composite (Tetric $\mathrm{N}$ Ceram, Ivoclar Vivadent) and fit onto the prepared teeth in this group (-Fig. 1). Excess materials (mostly observed in the buccal and lingual) were removed with a carver and with an 80-second curing time (20 second on each side of the tooth). The celluloid matrix was removed, and minor polishing was done. ${ }^{8}$

In groups $\mathrm{A}$ and $\mathrm{B}$, the crown designs were made using exocad $\mathrm{GmbH}$ software (GmbH, Darmstadt, Germany) after digitally scanning the prepared teeth using Medit T300 3D Dental Scanners (MEDIT Corp. 23 Goryeodae-ro 22-gil, Seongbuk-gu, Seoul, South Korea). The Exocad software program provides a database containing morphologic data on all known permanent tooth forms. This database was used in the creation of two indirect restorations used in groups $A$ and B. The similarity of permanent first molar and the primary second molar designs was referenced, and our testing used the same design ( - Fig. 2 ).

The restoration type for groups A and B was selected as a full crown with an average thickness of $1.5 \mathrm{~mm}$ at the central

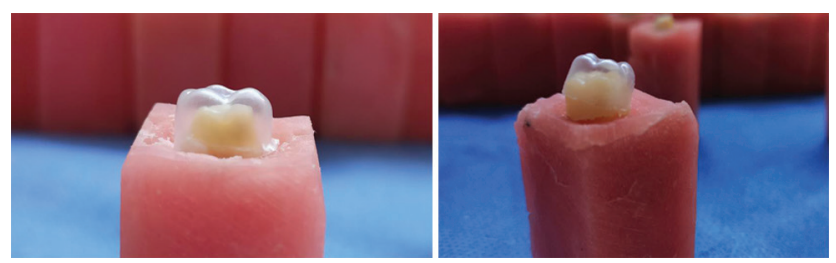

Fig. 1 Celluloid crowns placement. 

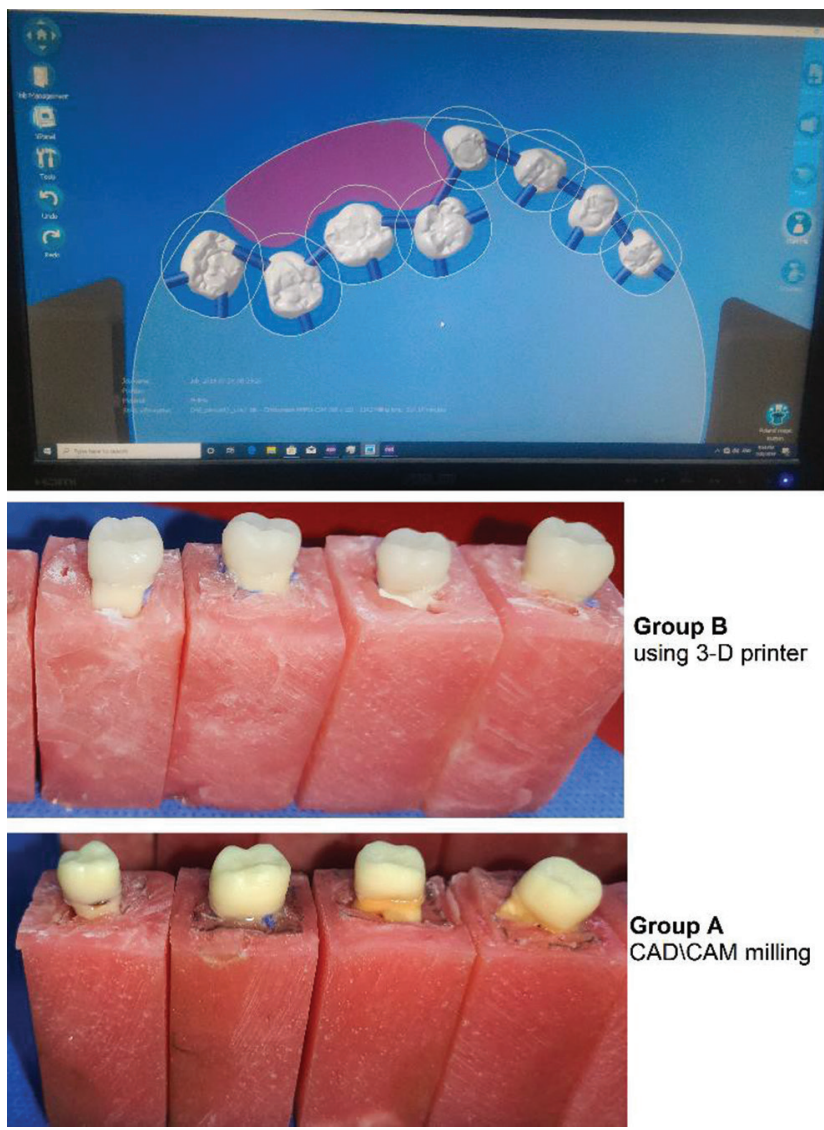

Fig. 2 exocad $\mathrm{GmbH}$ software and fabricated crowns in groups $A$ and $B$.

groove, and $1 \mathrm{~mm}$ on the axial surfaces with a maximum of $2.0 \mathrm{~mm}$ at the cusp. The STL files were generated for the 20 crowns in each group (A and $B$ ) and then fabricated according to their respective groups; group A using PMMA blocks (White Peaks Dental Solutions GmbH \& Co. KG Germany) and group $\mathrm{B}$ using 3D printable light-curing composite fabricated with a 3D dental printer (GC Temp PRINT, Tokyo, Japan). GC Temp PRINT is a biocompatible Class IIa (medium risk) printable material used for provisional crowns and bridges fabrication. It is free of methyl methacrylate (MMA) and is designed to be used for digital light processing-based 3D printing (-Fig. 3 ).

CopraTemp Symphony PMMA material is suitable for the fabrication of crowns and bridges that serve as a provisional or long-term dental prosthesis. It is also suitable for permanent prosthesis plaque resistance according to the manufacturer's provided information.

The crowns were tried on to ensure a passive fit, any noticeable undercuts in the prepared teeth were removed with a composite finishing bur. The crowns in groups $\mathrm{A}$ and B were cemented using Resin-cement (G-CEM LinkAce, GC Corporation, Tokyo, Japan), all cementation procedures followed the manufacturers' instructions, then the die-crown units were allowed to set for 24 hours. All samples were subsequently stored in distilled water for 30 days.

Fracture resistance for each crowned tooth in all three groups was performed with a universal mechanical testing

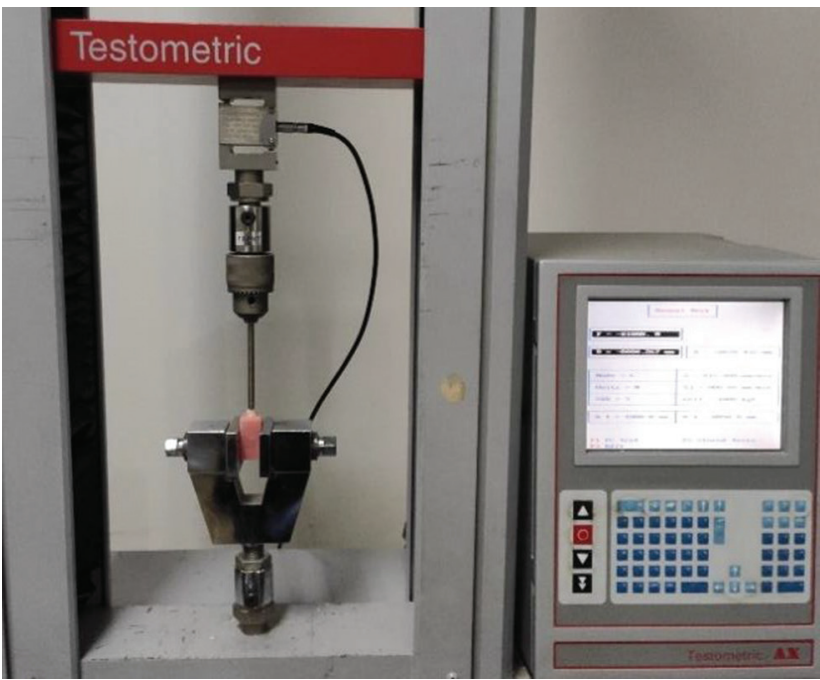

Fig. 3 Universal mechanical testing machine (Testometric).

machine (Testometric 50Kn, Co Ltd, UK), and loading was increased until the crown fractured..$^{16}$ The uniaxial force was delivered through a stainless-steel ball fixture to replicate a cusp contact.

Testing was performed in a single cycle, with the speed of the crosshead maintained at $1 \mathrm{~m} / \mathrm{min}$, until the experimented crowned teeth fractured ${ }^{17}$; the fractured teeth|crowns were photograph using Xiaomi mi9 phone camera.

Following the load-to-fracture tests, the specimens were analyzed for one of the three failure types: repairable crowns|teeth-fracture, repairable, or catastrophic tooth-root fracture. ${ }^{18}$ All three types of tested crowns were compared for the force required for fracture. The confidence intervals around the mean were also calculated and compared with a control value of 78 to $106 \mathrm{~N}$ for posterior occlusal loads, as reported previously by Braun et al ${ }^{19}$ ( - Table $\mathbf{1}$ ).

All analyses were performed using SPSS 20.0 for Windows (SPSS; Chicago, Illinois, United States).

Fracture types were compared using Chi-square test. The load-to-fracture scores obtained from three types of crowns were compared by using one-way analysis of variance test. The significance level was set at $p<0.05$.

\section{Results}

FRF is illustrated in - Table 1. One-way analysis of variance (ANOVA) ( - Table 2 ) revealed significant differences among the groups in FRF experimental testing $(F=20.584, p=0.000)$. The Bonferroni test (-Table 3 ) was used for multiple correction in the comparison of the force needed to fracture the specimens in the three groups. This test demonstrated significant differences between groups B and C $(p=0.000)$ and also, between groups $A$ and $C(p=0.000)$. However, there was no statistically significant difference between groups $A$ and B $(p=0.325)$ in the FRFs score (Graph 1).

The fracture types of the experimental crowns|teeth are presented in - Table 4, Graph 2, and illustrated in -Fig. 4. No statistical differences in fracture type were noticed when comparing groups A and B or groups B and C. However, a 
Table 1 Fracture resistance force between three groups

\begin{tabular}{|l|l|l|l|}
\hline Groups & $\mathbf{N}$ & Mean & Std. deviation \\
\hline Group A & 10 & 1,719 & 341.608 \\
\hline Group B & 10 & $1,494.05$ & 320.675 \\
\hline Group C & 10 & 879.51 & 235.981 \\
\hline
\end{tabular}

Table 2 One-way ANOVA to study the difference in the three groups in FRF

\begin{tabular}{|l|l|l|l|}
\hline Source & Degree of freedom & $F$ & $p$-Value \\
\hline Between the groups & 2 & 20.584 & 0.000 \\
\cline { 1 - 2 } Intragroup & 27 & & \\
\hline Total & 29 & \\
\hline
\end{tabular}

Abbreviation: FRF, fracture resistance force.

Table 3 Bonferroni test for multiple-correction comparison in FRF

\begin{tabular}{|l|l|l|l|l|}
\hline Groups & Source & $\begin{array}{l}\text { Mean } \\
\text { difference }\end{array}$ & $p$-Value & Decision \\
\hline Group C & Group B & $614.54^{\text {a }}$ & 0.000 & $\begin{array}{l}\text { Statistical differences toward } \\
\text { group B }\end{array}$ \\
\cline { 2 - 5 } & Group A & $839.49^{a}$ & 0.000 & $\begin{array}{l}\text { Statistical differences toward } \\
\text { group A }\end{array}$ \\
\hline Group B & Group A & 224.95 & 0.325 & No statistical differences \\
\hline
\end{tabular}

Abbreviation: FRF, fracture resistance force.

\section{The forces needed to fracture of specimens}

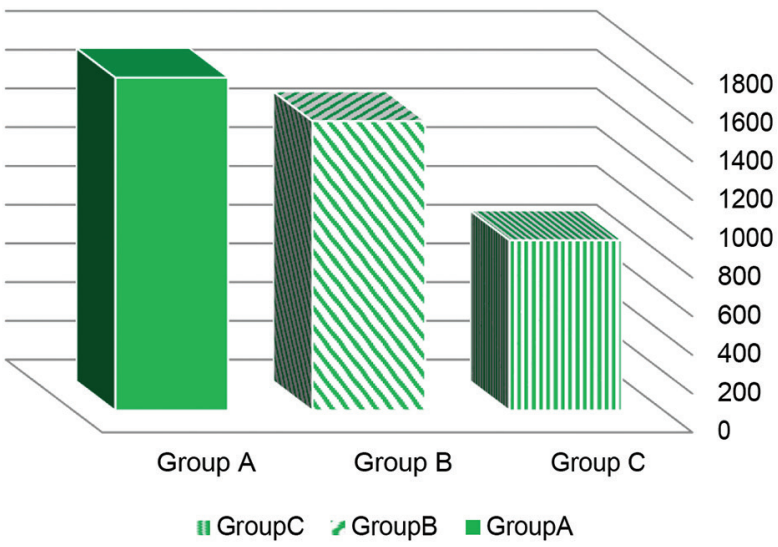

Graph 1 Fracture resistance for the crowned teeth in the three groups.

statistical difference was noticed between groups A and C when comparing fracture type among the three groups in the chi-square statistical test ( $\mathbf{- T a b l e ~} \mathbf{5}$ ).

As noticed in - Table 4 and Graph 2, the indirect crowns in groups A and B show a type of "Not Repairable" fracture type. Interestingly, crowns created with the direct technique in group $\mathrm{C}$ did not show any catastrophic failure. It was found that $30 \%$ of the samples from groups A and C showed repairable fracture types while $50 \%$ of crowned teeth in group B show repairable fracture types.

\section{Discussion}

The literature is filled with challenges in the management of pulpotomized primary molars with large cavity preparations requiring restoration with filling materials. These restored teeth with largely unsupported cusps result in fracture during function. To manage these types of situations clinicians will turn to full coverage restorations, typically SSCs. ${ }^{20}$ This has been the norm for many years.

The use of bonded restorations has dramatically evolved in recent years, and the demand for esthetic restorations has led to the development of diverse types of cosmetic materials. ${ }^{21}$ There is a need to know if these new materials will function in the oral cavity for pediatric patients and are comparable to traditional SSCs. We know that the replication of clinical conditions is not easily simulated by in vitro studies. Epoxy materials are often employed to simulate tooth structure in fracture resistance studies. However, these epoxy material test dies are unreliable and often create limitations fracture resistance studies. ${ }^{19}$ To our knowledge, no studies have been conducted to compare the FRF abilities of these materials when cemented or bonded to natural teeth. Therefore, this study used natural teeth and obtained data on the fracture resistance of these various provisional crowns to attempt to simulate the clinical situation..$^{22}$ The use of 3D printable photopolymer resin and comparing it to milled crowns and direct composite fabricated celluloid crowns was the novel aspect of this study. 3D printing has an enormous potential in the field of clinical dentistry, in almost every discipline, therefore understanding that application in 
Table 4 Failure type after load-to-failure testing

\begin{tabular}{|c|l|l|l|l|l|}
\hline \multirow{2}{*}{ Groups } & \multicolumn{2}{|c|}{ Repairable } & \multicolumn{2}{c|}{ Possibly repairable } & Not repairable \\
\cline { 2 - 6 } & $\begin{array}{l}\text { Cohesive } \\
\text { failure }\end{array}$ & $\begin{array}{l}\text { Adhesive } \\
\text { failure }\end{array}$ & $\begin{array}{l}\text { Adhesive failure }+ \\
\text { crack or chip } \\
\text { dentine }\end{array}$ & $\begin{array}{l}\text { Adhesive failure + } \\
\text { deep crack or chip } \\
\text { dentine }\end{array}$ & \begin{tabular}{l} 
Catastrophic failure \\
\hline Group A
\end{tabular} \\
\hline Group B & 1 & 3 & 2 & 3 & 2 \\
\hline Group C & 2 & 4 & 3 & 5 & 1 \\
\hline$\%$ & $36.7 \%$ & 1 & 2 & $53.3 \%$ & $10 \%$ \\
\hline
\end{tabular}

\section{Failure type after load-to-failuretest}

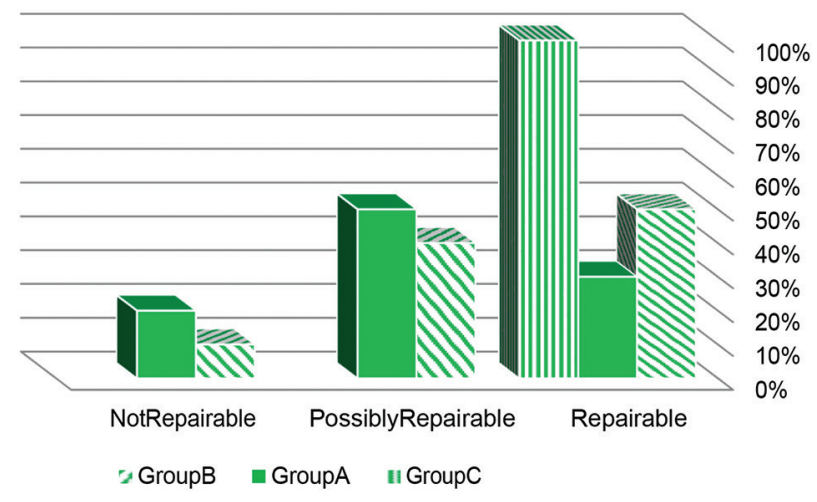

Graph 2 Failure type after the load-to-failure test.

the restoration of pulpotomized primary molars in pediatric dentistry can offer another dimension to the management of the primary dentition. ${ }^{23}$

The mean maximum bite force in 6 to 8 years old is $76 \mathrm{~N}$ and $106 \mathrm{~N}$ in 10 to 12 year old in the primary molars region..$^{24,25}$ The mean force values needed to fracture the crowns fabricated in the 3D printable photopolymer resin group were $1,494.05 \mathrm{~N}$, $1,719 \mathrm{~N}$ in the CAD|CAM group, and $879.51 \mathrm{~N}$ in direct composite using celluloid crown group. Therefore, the mean force to fracture the experimental crowns in this study exceeded these reported mean maximum bite force values, and exhibited higher fracture resistance values than the average bite force.

On the basis of this study, we have demonstrated that printable photopolymer resin (GC Temp PRINT) restorative dental material, via a 3D printing system are able generate long-term provisional restorations acceptable for intraoral use with sufficient mechanical properties. ${ }^{12}$

Additionally, as expected, the crowns fabricated from PMMA blocks using CAD $\mid C A M$ showed higher fracture resistance than the other two groups (3D-printable resin or direct composite using celluloid crowns). This is due to the fact that these milled blocks are created via high pressure/ high-temperature polymerization, resulting in a reduction in the number and size of defects that led to the improvement of the fracture toughness. ${ }^{26}$
The failure type of direct composite celluloid crowns in group C demonstrated "Repairable|Possibly Repairable" fracture types with no Catastrophic Failure, as compared with groups A and B. The direct technique is more sensitive technique because of the direct bonding process and intraoral moisture. Nevertheless, this procedure is easy to repair using typical composite resin as compared with the indirect techniques used in this study. ${ }^{26}$ The failure type of the indirect crowns tested showed a 10\% (group B) and 20\% (group A) "not repairable" fracture type (Graph 2 ).

Within the limitations of this study, it was determined that all three types of the esthetic provisional crowns tested, exceeded the mean maximum bite for of children in primary dentition. The limitations of this study arise from (1) the use of natural extracted teeth, (2) the film thickness of the cement, and (3) the differences in the size of these molars. The exocad $\mathrm{GmbH}$ software ( $\mathrm{GmbH}$, Darmstadt, Germany) was used to in an attempt to overcome this and generate a uniform thickness at the interface of the tooth and the intaglio surface of the crowns cemented to the prepared teeth in groups A and B.

Another potential limitation for this study, is that the force required to fracture the crowns in vivo may have been underestimated in this study and higher values for force required to fracture the crowns may have been observed if the dies were restored with different types of restoring materials (such as GIC cements, RMGIC filling materials).

\section{Conclusion}

This study provides evidence that all three types of esthetic provisional crowns tested have clinical applicability. There were significant differences in fractures resistance among the three experimental groups, with 3D printable crowns and CAD $\mid C A M$ fabricated crowns showing a significantly higher fractured resistance force compared with direct celluloid composite crowns. The indirect technique (CAD $\mid C A M$ milling and 3D printer) showed a higher score in FRF. However, the direct composite celluloid crowns technique is easier to repair when compared with indirect techniques in failure type. 

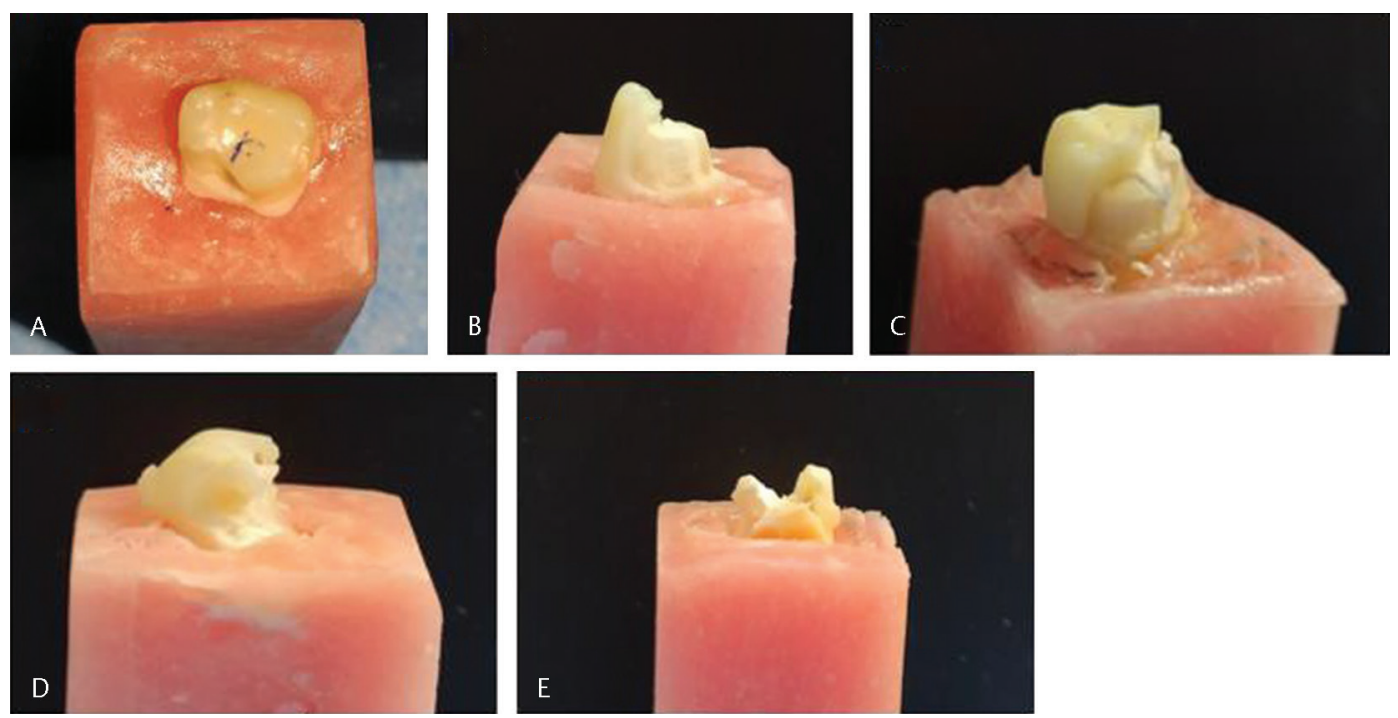

Fig. 4 Failure type after load-to-failure testing graphic definitions. (A) Cohesive failure. (B) Adhesive failure, (A, B) "Restorable," (C) Adhesive failure and chip or crack dentin. (D) Adhesive failure and deep chip or crack dentin, (C, D) "Possibly repairable," (E) Catastrophic failure, "not repairable."

Table 5 Chi-square test-failure type comparisons

\begin{tabular}{|l|l|l|l|}
\hline Material group comparisons & Chi-square & $p$-Value & Decision \\
\hline Group A-B & 0.117 & 0.117 & No statistical differences \\
\hline Group A-C & 10.0 & 0.007 & $\begin{array}{l}\text { Statistical differences } \\
\text { toward } \\
\text { group C }\end{array}$ \\
\hline Group B-C & & & No statistical differences \\
\hline
\end{tabular}

\section{Authors' Contributions}

All the authors took part in conducting the study, manuscript preparation, and editing.

\section{Conflict of Interest}

None declared.

\section{Acknowledgment}

This study has been performed at the Dental College at Damascus University, which deserves a great appreciation for all academic and financial support.

\section{References}

1 Zimmerman JA, Feigal RJ, Till MJ, Hodges JS. Parental attitudes on restorative materials as factors influencing current use in pediatric dentistry. Pediatr Dent 2009;31(1):63-70

2 Fishman R, Guelmann M, Bimstein E. Children's selection of posterior restorative materials. J Clin Pediatr Dent 2006; 31(1):1-4

3 Roberts JF. The open-face stainless steel crown for primary molars. ASDC J Dent Child 1983;50(4):262-263

4 Kindelan SA, Day P, Nichol R, Willmott N, Fayle SA; British Society of Paediatric Dentistry. UK National Clinical Guidelines in Paediatric Dentistry: stainless steel preformed crowns for primary molars. Int J Paediatr Dent 2008;18(suppl 1) :20-28

5 Attari N, Roberts JF. Restoration of primary teeth with crowns: a systematic review of the literature. Eur Arch Paediatr Dent 2006;7(2):58-62, discussion 63

6 Innes NP, Ricketts DN, Evans DJ. Preformed metal crowns for decayed primary molar teeth. Cochrane Database Syst Rev 2007;(1):CD005512
7 Terry DA, Leinfelder KF, Maragos C. Developing form, function, and natural aesthetics with laboratory-processed composite resin-part II. Pract Proced Aesthet Dent 2005;17(7):449-454, quiz 456

8 Ram D, Peretz B. Composite crown-form crowns for severely decayed primary molars: a technique for restoring function and esthetics. J Clin Pediatr Dent 2000;24(4):257-260

9 Mainjot AK, Dupont NM, Oudkerk JC, Dewael TY, Sadoun MJ. From artisanal to CAD-CAM blocks: state of the art of indirect composites. J Dent Res 2016;95(5):487-495

10 Frazier WE. Metal additive manufacturing: a review. J Mater Eng Perform 2014;23(6):1917-1928 doi:10.31399/ asm.hb.v20. a0009211

11 Lee W-S, Lee D-H, Lee K-B. Evaluation of internal fit of interim crown fabricated with CAD/CAM milling and 3D printing system. J Adv Prosthodont 2017;9(4):265-270

12 Tahayeri A, Morgan M, Fugolin AP, et al. 3D printed versus conventionally cured provisional crown and bridge dental materials. Dent Mater 2018;34(2):192-200

13 Syed M, Chopra R, Sachdev V. Allergic reactions to dental materials-a systematic review. J Clin Diagn Res 2015;9(10): ZE04-ZE09

14 Giordano R. Materials for chairside CAD/CAM-produced restorations. J Am Dent Assoc 2006;137(suppl) :14S-21S

15 Mourouzis P, Arhakis A, Tolidis K. Computer-aided design and manufacturing crown on primary molars: an innovative case report. Int J Clin Pediatr Dent 2019;12(1):76-79

16 Magne P, Knezevic A. Influence of overlay restorative materials and load cusps on the fatigue resistance of endodontically treated molars. Quintessence Int 2009;40(9):729-737

17 Townsend JA, Knoell P, Yu Q, et al. In vitro fracture resistance of three commercially available zirconia crowns for primary molars. Pediatr Dent 2014;36(5):125-129 
18 Carvalho AO, Bruzi G, Giannini M, Magne P. Fatigue resistance of CAD/CAM complete crowns with a simplified cementation process. J Prosthet Dent 2014;111(4):310-317

19 Braun S, Hnat WP, Freudenthaler JW, Marcotte MR, Hönigle K, Johnson BE. A study of maximum bite force during growth and development. Angle Orthod 1996;66(4):261-264

20 el-Kalla IH, García-Godoy F. Fracture strength of adhesively restored pulpotomized primary molars. ASDC J Dent Child 1999;66(4):238-242, 228

21 Omer H. Fracture resistance of puplotomized primary molars restored with various restorative materials. Acta Scientific Dent Sci 2019;3:98-104

22 Magne P, Carvalho AO, Bruzi G, Giannini M. Fatigue resistance of ultrathin CAD/CAM complete crowns with a simplified cementation process. J Prosthet Dent 2015;114(4):574-579
23 Salmi M, Paloheimo K-S, Tuomi J, Ingman T, Mäkitie A. A digital process for additive manufacturing of occlusal splints: a clinical pilot study. J R Soc Interface 2013;10(84):20130203

24 Gavião MBD, Raymundo VG, Rentes AM. Masticatory performance and bite force in children with primary dentition. Braz Oral Res 2007;21(2):146-152

25 Braun S, Hnat WP, Freudenthaler JW, Marcotte MR, Hönigle K, Johnson BE. A study of maximum bite force during growth and development. Angle Orthod 1996;66(4):261-264

26 Nguyen J-F, Migonney V, Ruse ND, Sadoun M. Resin composite blocks via high-pressure high-temperature polymerization. Dent Mater 2012;28(5):529-534 